\title{
Gallstone ileus: a new role for abdominal computed tomography
}

\author{
Eric CJ Seal, Michael F Creagh, Peter J Finch
}

\begin{abstract}
Summary
This case report illustrates the difficulties in making the clinical diagnosis of gallstone ileus. In addition to an unusual clinical picture, the changes normally expected on abdominal $\mathrm{X}$-ray in gallstone ileus were subtle and missed in our patient. The diagnosis was made using abdominal computerised tomography (CT). We recommend the early use of abdominal CT scanning in the investigation of clinical bowel obstruction, especially in the elderly, where gallstone ileus is a more common condition.
\end{abstract}

Keywords: gallstone ileus, computed tomography, diagnosis

Gallstone ileus is an uncommon cause of bowel obstruction. The diagnosis is usually made on the basis of the characteristic abdominal X-ray appearance, or less commonly, from abdominal ultrasound, or upper abdominal contrast studies. In this paper, we present a patient where gallstone ileus was diagnosed following abdominal CT scan.

\section{Case report}

A 69-year-old woman presented to hospital with a two-month history of abdominal pain and diarrhoea, which was of sudden onset. Vomiting, although prominent initially, had settled by the time of presentation. Pain was not a feature, but she did complain of anorexia, with an associated weight loss of $12 \mathrm{~kg}$. Her stools were semiformed and pale. There was no blood or mucus.

She had no relevant past medical history, was taking no medications, and had no allergies. She was a non-smoker, and rarely took alcohol.

At presentation, she was emaciated. Her abdomen was soft, and a mobile mass was palpable in the right iliac fossa, measuring $5 \mathrm{~cm}$ in diameter. The right side was distended. Rectal examination was unremarkable. On investigation she was mildly anaemic. The results of the biochemical investigations are given in the box.

Plain abdominal X-ray showed possible small bowel obstruction, with a distended loop of small bowel on the right of the spine, and thickened valvulae conniventes. Multiple loops were fluid filled, and very little gas was evident in the colon (figure 1).
The presumptive diagnosis was carcinoma of the caecum causing obstruction, with hepatic metastases, and possible bony secondaries, on the basis of a disproportionately high alkaline phosphatase. She was admitted to the ward for further investigation.

Ultrasound offered no further information. No pelvic mass was identified, and liver and kidneys were both normal. Fluid-filled loops of

\begin{tabular}{|ll|}
\hline \multicolumn{2}{|l|}{ Biochemical investigations } \\
\hline & Value (normal \\
& range) \\
Investigation & 10.1 \\
haemoglobin $(\mathrm{g} / \mathrm{dl})$ & $81.3(80-96)$ \\
mean corpuscular volume $(\mathrm{fl})$ & 7.3 \\
white cell count & 400 \\
platelet count & $65(0-40)$ \\
alanine transferase (IU/l) & $1207(80-300)$ \\
alkaline phosphatase (IU/l) & $12(2-19)$ \\
bilirubin $(\mu$ mol/l) & $139(<50)$ \\
$\gamma$-glutamyl transferase $(\mathrm{IU} / \mathrm{l})$ & $170(320-490)$ \\
albumin $(\mathrm{g} / \mathrm{l})$ & \\
\end{tabular}

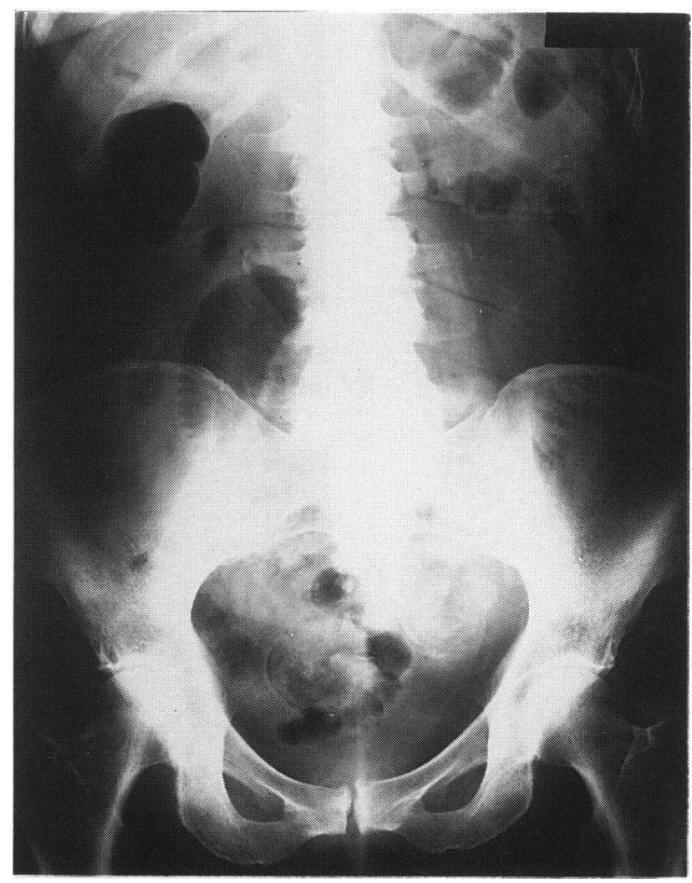

Figure 1 Plain abdominal X-ray, reporting the possibility of possible small bowel obstruction. Note, however, the appearance of stone in the pelvis and also the presence of gas in the gall bladder 
bowel were the only prominent feature. The gall bladder and biliary tree were poorly visualised. Double-contrast barium enema failed to show any colonic lesions. The caecal pole was distended, and there was no reflux of contrast into the terminal ileum. Two isolated sigmoid diverticula were noted.

Her condition deteriorated in hospital. The haemoglobin fell to $9.3 \mathrm{~g} / \mathrm{dl}$ and she was transfused. Vomiting had again become prominent, and she underwent gastroscopy. This demonstrated a fluid-filled stomach, and an oedematous but functioning pylorus. Bone scan was also performed in view of the high alkaline phosphatase, and was normal. Total parenteral nutrition was commenced.

Abdominal CT scan was performed 14 days after admission, as it took eight days for the barium to clear from the colon following the enema. From this investigation the unexpected diagnosis of gallstone ileus was made. Gas was observed within the biliary tree, and within the contracted gall bladder (figure 2). The stomach and small bowel were distended with fluid. An intraluminal mass was also seen in the right iliac fossa, consistent with a single stone impacting the small bowel (figure 3).

She was referred for surgery. At laparatomy, a large gallstone impacting the proximal jejunum was extracted (figure 4). A large inflammatory mass with shrunken gall bladder marked the presence of a cholangio-duodenal fistula. No attempt was made to close this.

Post-operatively, her recovery was slow. Anaemia persisted, requiring further trans-

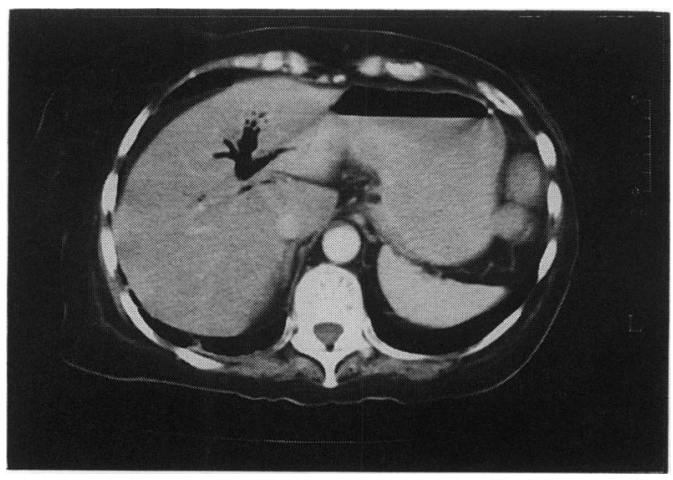

Figure 2 Abdominal CT scan showing gas within the intrahepatic biliary system

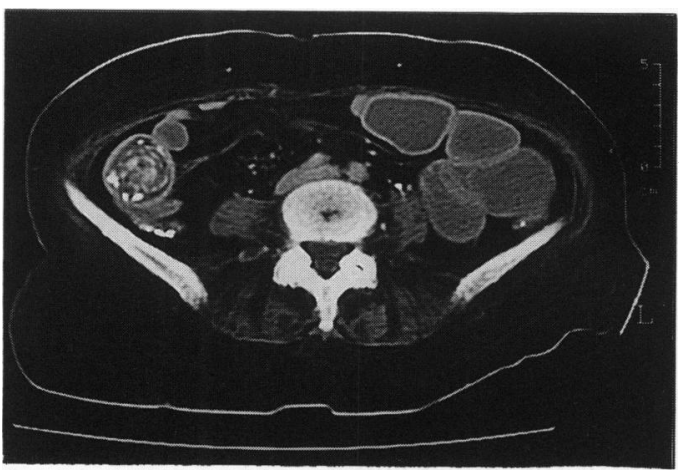

Figure 3 A 4-cm calculus is demonstrated in the right iliac fossa on CT scan. Dilated loops of bowel filled with fluid are also seen fusion. Endoscopic retrograde cholangiopancreatography (ERCP) was performed in view of persistently raised liver function tests. The biliary system was distended and a $1-\mathrm{cm}$ calculus was demonstrated in the gall bladder. Small debris was also observed in the common bile duct. Sphincterotomy was performed, and the debris was removed. During this convalescent phase she was also treated with parenteral, then oral, antibiotics.

Poor general condition at presentation prolonged the period of hospitalisation. Eventually she recommenced and tolerated solids, she slowly mobilised and was discharged after 10 weeks in hospital.

\section{Discussion}

In our patient diagnosis was delayed until abdominal CT scan eventually confirmed the presence of gallstone ileus. In retrospect the diagnosis could have been made on the day of presentation on the basis of the abdominal $\mathrm{X}$-ray appearance.

The classical signs on X-ray of gallstone ileus were described in 1941 by Rigler et al (see box). ${ }^{1}$ Abdominal ultrasound is also said to be useful in diagnosis, ${ }^{2}$ but was unhelpful in our patient.

Recent reports have advocated the use of early abdominal CT scanning in the diagnosis of gallstone ileus. ${ }^{3,4}$ The characteristic radiological changes of gallstone ileus are easily discernible on CT. ${ }^{4}$ If CT scan had been performed sooner in our patient the delay to surgery would have been considerably less, and a number of investigations possibly avoided.

Gallstone ileus is the cause of $2 \%$ of all mechanical bowel obstructions, ${ }^{2,5}$ hence, it may not readily spring to mind as a diagnosis. The incidence increases in elderly patients, however, ${ }^{5,6}$ with one series reporting gallstones to

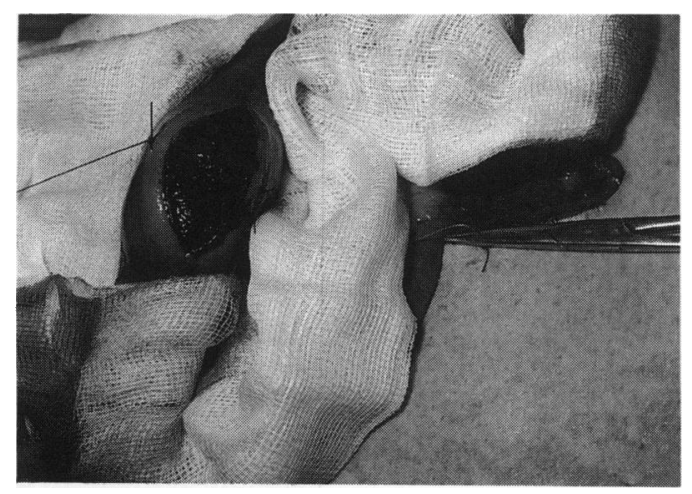

Figure 4 Jejunotomy and stone removal at laparotomy

Gallstone ileus: radiological features

- pneumobilia

- mechanical bowel obstruction

- presence of a new stone, or change in position of an existing one 


\section{Gallstone ileus: clinical features}

- more common in the elderly

- prior biliary disease noted in almost half the cases

- abdominal pain prominent

- intermittent mechanical bowel obstruction related to 'tumbling' phenomenon

- associated medical illness common (especially diabetes and cardiovascular disease)

be the cause of obstruction in $25 \%$ of patients over the age of 65 years. ${ }^{7}$

The common symptoms of gallstone ileus are listed in the box. The presence of biliary symptoms before the onset of bowel obstruction is said to be as high as $50 \% \cdot{ }^{10}$ In a series of 34 cases reviewed by Day and Marks, the incidence of biliary symptoms prior to presentation was $38 \%^{8}$

Another clinical feature is the 'tumbling' phenomenon. The usual presentation of gallstone ileus is of intermittent episodes of partial or complete bowel obstruction. These episodes correspond to stone impaction and subsequent release. The stone then 'tumbles' down the lumen only to impact again more distally. ${ }^{9}$ This did not occur in our patient, as the stone was discovered impacted in the proximal jejunum. Presumably, its size would not allow disimpaction.

The mortality and morbidity of gallstone ileus is high. In some series death occurs in as many as $20 \%$ of patients. ${ }^{8,11}$ This is probably related to the fact that the bowel obstruction occurs in an older age, a group of patients with other associated medical problems. ${ }^{8,9}$

1 Rigler LG, Borman CN, Noble JF. Gallstone obstruction; pathogenesis and roentgen manifestations. F $A M A$ 1941; 117: 1753-9.

2 Davies RJ, Sandrasagra FA, Joseph AE. Case report: ultrasound in the diagnosis of gallstone ileus. Clin Radiol 1991; 43: 282-4

3 Loren I, Lasson A, Nilsson A, Nirhov N. Gallstone ileus Loren I, Lasson A, Nilsson A, Nirhov N. Gallstone ileus
demonstrated by CT. $\mathcal{F}$ Comput Assist Tomogr 1994; 18: $262-5$

4 Leen GLS, Finlay M. CT diagnosis of gallstone ileus. Acta Radiol 1990; 31: 497-8.

5 Kasahara Y, Umemura H, Shiraha S, Kuyama T, Sakata K, Kubota $H$. Gallstone ileus: review of 112 patients in the Japanese literature. Am $\mathcal{f}$ Surg 1980; 140: 437-40.

6 Gajraj H. Gallstone ileus (letter). Br F Surg 1991; 78: 378.

7 Moss JF, Bloom AD, Mesleh GF, Deziel D, Hopkins WM. Gallstone ileus. Am Surg 1987; 53: 424-8.
The treatment of choice for gallstone ileus is surgery. The correct surgical option remains controversial. Current literature favours enterolithotomy only. The surgeon then has the option of definitive biliary surgery later if symptoms persist. ${ }^{5,10,12}$ This was the approach in our patient. Indeed, she did continue to remain unwell following surgery, but both symptoms and liver function improved following sphincterotomy.

The alternative to enterolithotomy alone is a combined procedure of stone extraction associated with cholecystectomy. The rationale for this approach is that the combined procedure prevents the complications of recurrent gallstone ileus, cholangitis, and carcinoma of the gall bladder. These complications are said to occur in $30 \%$ of patients treated with enterolithotomy alone. ${ }^{13}$

\section{Conclusion}

Gallstone ileus is a serious disease. Although not a common cause of mechanical bowel obstruction, the incidence increases with age, and should be suspected in the elderly. Radiological signs on plain abdominal X-ray may be subtle and easily missed. Abdominal CT scan allowed the correct diagnosis to be made in our patient. Hence we would recommend the use of early CT scanning in patients with unexplained bowel obstruction, especially the elderly.

Special thanks to Mr Graham Layer, Consultant Surgeon, St Peter's Hospital, for his assistance in the preparation of this manuscript.

8 Day E, Marks C. Gallstone ileus: review of the literature and presentation of thirty-four new cases. Am ₹ Surg 1975; 129: p52-8.

9 VanLandingham SB, Broders CW. Gallstone ileus. Surg Clin N Am 1982; 62: 241-7.

10 Clavien PA, Richon J, Burgan S, Rohner A. Gallstone ileus. Clavien PA, Richon J, Burgan
Br $\mathcal{F}$ Surg 1990; 77: 737-42.

11 Deitz DM, Standage BA, Pinson CW, McConnell DB Krippaehne WW. Improving the outcome in gallstone ileus Am $\mathcal{F}$ Surg 1986; 151: 572-6.

12 Syme RG. Management of gallstone ileus. Can f Surg 1989, 32: $61-4$.

13 Kirkland KC, Croce EJ. Gallstone intestinal obstruction. A review of the literature and presentation of 12 cases including 3 recurrences. $\mathcal{F} A M A$ 1961; 176: 494-7. 